\title{
INCIDENCE OF SUBCLINICAL NEUROPATHY IN NEWLY DIAGNOSED HIV POSITIVE PATIENTS
}

\author{
Veena Narisetty1, Dhairyawan Pokalkar ${ }^{2}$ \\ ${ }^{1}$ Assistant Professor, Department of Neurology, Gandhi Medical College, Hyderabad, Telangana. \\ 2Professor, Department of Neurology, Gandhi Medical College, Hyderabad, Telangana.
}

ABSTRACT
BACKGROUND
About $10-20 \%$ of HIV seropositive subjects present with neurological involvement and $50-70 \%$ have evidence of neurological
involvement during the course of illness. Autopsy studies showed nervous system involvement in $80-90 \%$ of subjects. The
commonest neurological complication is peripheral neuropathy, the Distal Symmetrical Sensory Neuropathy (DSP).

\section{METHODS}

We have conducted a prospective hospital based study to know the incidence of subclinical neuropathy in asymptomatic newly diagnosed HIV patients in the Department of Neurology, Gandhi Medical College, Secunderabad, Telangana state, a tertiary care teaching hospital. The study group was selected from the patient pool attending the regional Antiretroviral Therapy (ART) Clinic at Gandhi Hospital from 2012 to 2015. These patients do not have any symptoms suggestive of peripheral nerve involvement. However, clinical examination was abnormal in $40 \%$ of patients. These patients were not on ART drugs or do not have any chronic medical illness or hereditary or nutritional cause for neuropathy. Asymptomatic patients seropositive for HIV infection attending Gandhi Hospital from 2012 to 2015 were screened for evidence of neuropathy. Patients on antiretroviral treatment were excluded. Specific investigations were done to exclude other causes of neuropathy.

\section{RESULTS}

In our study of $200 \mathrm{HIV}$ positive asymptomatic patients, the nerve conduction studies were normal in 62 patients. The other 128 patients have various abnormalities in nerve conduction studies in the form of asymptomatic sensory axonopathy, predominantly affecting the lower limbs (length dependent) in 54 patients, motor mononeuropathy and multiple mononeuropathies in 42 patients and sensorimotor demyelinating and axonal neuropathy in 42 patients.

\section{CONCLUSIONS}

The present study shows evidence of subclinical neuropathy in almost $69 \%$ of patients.

\section{KEYWORDS}

Distal Symmetrical Polyneuropathy, Neuropathy, Human Immunodeficiency Virus, Anti-Retroviral Therapy, Acquired Immune Deficiency Syndrome.

HOW TO CITE THIS ARTICLE: Narisetty V, Pokalkar D. Incidence of subclinical neuropathy in newly diagnosed HIV positive patients. J. Evolution Med. Dent. Sci. 2016;5(62):4342-4345, DOI: 10.14260/jemds/2016/991

\section{INTRODUCTION \\ HIV infection is a global pandemic affecting 36.9 million(1) people (2014 WHO estimate) across the world of which 2.1 million(2) are living in Indian subcontinent. Approximately, the number of asymptomatic HIV seropositive is three times of those with the Acquired Immunodeficiency Syndrome (AIDS). \\ Peripheral neuropathies are common in HIV infection complicating all stages of HIV disease and cause considerable morbidity and disability in HIV infected individuals and AIDS patients.(3,4) Although symptomatic neuropathy occurs in 10- $15 \%$ of HIV infected individuals, overall pathological evidence of peripheral nerve involvement is present in virtually all end-stage AIDS patients.(5)}

Financial or Other, Competing Interest: None

Submission 12-07-2016, Peer Review 25-07-2016,

Acceptance 27-07-2016, Published 02-08-2016.

Corresponding Author:

Dr. Veena Narisetty,

Assistant Professor,

Department of Neurology,

Gandhi Medical College,

Hyderabad,

Telangana.

E-mail: veenanarisetty@yahoo.com

DOI: $10.14260 /$ jemds/2016/991
There are Four Major Clinical types of HIV Associated Neuropathies that are regularly seen in Large HIV Clinics

a. Distal Sensory Polyneuropathy (DSP).

b. Multiple mononeuropathies or isolated mononeuritis.

c. Inflammatory demyelinating polyradiculopathy.

d. CMV associated progressive lumbosacral polyradiculopathy.

Distal Symmetrical Polyneuropathy (DSP) is the commonest type of peripheral neuropathy present in up to $35 \%$ of patients with HIV infection.(6) which may occur secondary to HIV (HIV-DSP) or be due to antiretroviral drug toxicity (ARV-DSP). The clinical features of HIV-DSP and ARVDSP are identical. Asymptomatic DSP (Signs of peripheral nerve dysfunction without subjective pain) may occur in more than $50 \%$ of patients with less advanced disease ${ }^{(7)}$ and can be demonstrated by abnormal nerve conduction studies (NCS).(8)

Distal painful dysesthesias, allodynia, severe burning pain, pins and needles or insect crawling like sensations and numbness are the main symptoms. These symptoms usually begin in the feet, on the soles and progress up the legs. In more severe cases, symptoms may reach the knee or involve the hands, assuming the classic stocking and glove distribution. Formal neurological testing reveals evidence of 
abnormal distal reflexes most often reflected as reduced or absent ankle jerks bilaterally and sensory modalities in the form of impaired distal vibration sense and impaired pinprick sensation in a gradient manner like a stocking distribution. Motor examination is usually normal unless proximal large fiber nerves are affected.

Nerve Conduction Studies (NCS) show decreased conduction velocities and decreased amplitude of sensory nerve action potential, reduced compound motor action potential amplitudes and prolonged distal latencies and abnormalities in F waves. (9) NCS can be normal due to relatively low sensitivity for the detection of small fiber neuropathy. ${ }^{(10)}$

In the pre-ART era, studies suggested incidence rates of DSP as high as 89\%. The main risk factors for DSP were those related to advanced disease such as low CD4 T cell count and high plasma HIV viral load.(11,12) However, in the current HAART era, the correlation of these risk factors with DSP are less clear and HIV DSP was found to occur at higher CD4 T cell levels than previously observed and also in the context of undetectable plasma viral load.(13)

The currently observed increasing trend of HIV DSP is likely to reflect interrelated factors including increased life expectancy, age and life time exposure to antiretroviral, nutritional deficiency, use of neurotoxic drugs, alcohol exposure.(14) Other possible contributors include coinfection with Hepatitis C Virus (HCV), which has been associated with peripheral nerve disease and glucose intolerance with lipodystrophy.(15) Low baseline epidermal nerve fibre density $(<11 \mathrm{fibres} / \mathrm{mm})$ in skin biopsy in these patients correlates with the development of DSP. Mitochondrial dysfunction due to inhibition of mitochondrial DNA polymerase( ${ }^{(16)}$, neurotoxic effects of cytokines, effects of malnutrition and wasting on peripheral nerves, infections (CMV infection in addition) and some other indirect and unknown pathogenetic mechanisms were proposed to explain the cause of DSP.

We have conducted a prospective hospital-based study to know the incidence of subclinical neuropathy in asymptomatic newly diagnosed HIV patients who are not on ART drugs.

\section{AIM OF THE STUDY}

To know the incidence of subclinical neuropathy in newly diagnosed asymptomatic HIV positive patients.

\section{MATERIALS AND METHODS \\ Study Design}

The study group comprised of two hundred HIV seropositive patients who were newly diagnosed with HIV infection according to NACO guidelines, selected from the pool of patients who have attended Neurology OPD and AntiRetroviral Therapy (ART) Centre of Gandhi Hospital, between October 2012 and November 2015. After obtaining informed consent, only asymptomatic HIV subjects were included in the study. All the patients were evaluated within one month of diagnosis as HIV seropositivity. None of them were on antiretroviral drugs nor did they have any neurological or psychiatric illnesses that would interfere with the study. They did not have history of alcohol or drug abuse.

Demographic data regarding age and gender were obtained. Medical history regarding hypertension, diabetes mellitus, cardiac disease, smoking, alcoholism and family history of Neuropathy were recorded.

\section{The following Exclusion Criteria were Adapted in Selection of the Patients for the Study}

1. Patients on HAART therapy.

2. Patients exposed to neurotoxins like alcohol, isoniazid.(17) etc.

3. Medical illness other than HIV infection known to be associated with peripheral neuropathy. (Such as Diabetes mellitus, uraemia or chronic renal disease, chronic liver disease).

4. Family history of peripheral neuropathy.

Demographic data regarding age and gender were obtained. Medical History regarding hypertension, diabetes mellitus, cardiac disease, smoking, alcoholism and family history of Neuropathy were recorded.

Control population was derived from age, sex and education matched normal healthy volunteers.

Ethical clearance and standard protocols for research of the Institute were followed.

Detailed history was taken and neurological examination was done in all patients.

Routine biochemical tests like Complete Blood Picture (CBP), Blood sugar, BUN, Serum creatinine, Serum electrolytes, LFTs, Thyroid profile, Serum B12 estimation and CD4 counts were done.

A Nicolet EMG Machine and surface electrodes were used. Stimulation frequency was $1 \mathrm{~Hz}$ and duration was 200 microsec. Filters were set with high frequency at $2 \mathrm{kHz}$ and lower at $20 \mathrm{~Hz}$. The electrophysiology lab values were standardised. Routine motor conductions of median, ulnar, Common Peroneal (CPN) and Posterior Tibial (PTN) Nerve were performed. Antidromic sensory conductions of sural, median and ulnar sensory action potentials (SNAP) were recorded. Evidence of neuropathy was considered as per Ho et al criteria (Table 1). The data was computed for statistical analysis.

\section{RESULTS}

Among the total study population of two hundred patients $(n=200)$, there were 56\% (112) were men and 44\% (88) were women with male-female ratio of $5: 4$. There were $78 \%$ (156) patients between $20-40$ years of age. Remaining $22 \%$ (44) patients were above 40 years of age.

Clinical examination was abnormal in $40 \%$ (80) patients. Twenty four (12\%) patients had absent ankle reflexes. Twenty (10\%) patients had impaired pin prick and touch sensation up to knees and thirty six (18\%) patients had impaired vibration sensation distally.

Routine biochemical tests like CBP, blood sugar, BUN, serum electrolytes, LFTs, serum creatinine, thyroid function tests and serum B12 levels were all normal. Forty eight (24\%) patients have CD4 less than 200 (least CD4 value was $54)$.

Electrophysiological studies were abnormal in 69\% (138) patients.

Sensory conduction studies were abnormal in $27 \%$ (54) of patients. All SNAPs were lost in 12\% (24) patients. Isolated sural SNAP was lost in 8\% (16) of patients. Fourteen patients have loss of more than one SNAP in variable combinations. 
Motor conduction studies have shown demyelinating changes in CPN and PTN nerves in 22 (11\%) patients. Mononeuropathy of axonal type was seen in 28 (14\%). Multiple mononeuropathies in various combinations involving two or more nerves is seen in 14 (7\%) patients.

Combination of sensory and motor demyelinating and axonal changes were seen in 42 (21\%) of patients.

Prolongation of minimum $\mathrm{F}$ wave latencies were seen in $30(15 \%)$ patients. Absent $\mathrm{F}$ waves were seen in ten $(5 \%)$ patients.

\section{Table 1: Electrophysiological Criteria, Ho et al}

\section{A. Diagnosis of Demyelination \\ Present in Two or More Nerves}

1. Conduction velocity $<90 \%$ of lower limit normal if amplitude is $>50 \%$ of lower limit normal; $<85 \%$ if amplitude $<50 \%$ of lower limit of normal.

2. Distal latency $>110 \%$ of upper limit of normal if amplitude normal; $>120 \%$ of upper limit of normal, if amplitude is less than lower limit of normal.

3. Evidence of unequivocal temporal dispersion.

4. F-latency $>120 \%$ of normal.

\section{B. Diagnosis of Primary Axonopathy}

1. No evidence of demyelination as above.

2. Decrease in CMAP (Compound Muscle Action Potential) to $<80 \%$ of lower limit of normal, or

3. Denervation changes on needle electromyography.

\section{DISCUSSION}

Nervous system involvement is frequently seen in HIV patients adding to their morbidity and disabilities. DSP is the most common neurologic complication of HIV, affecting as many as $35 \%$ to $50 \%$ of patients according to various studies. Systematic electrophysiological testing had shown abnormalities in almost two-thirds of HIV positive individuals who did not have any symptoms of neuropathy.

In our study of 200 HIV positive asymptomatic patients, the nerve conduction studies were normal in 31\% (62) patients. The other $69 \%$ (138) patients have various abnormalities in nerve conduction studies in the form of asymptomatic sensory axonopathy predominantly affecting the lower limbs in $27 \%$ (54) patients, motor mononeuropathy and multiple mononeuropathies in $21 \%$ (42) patients and sensorimotor demyelinating and axonal neuropathy in $21 \%$ (42) patients. Electrophysiological study $(69 \%)$ has a higher diagnostic yield than clinical examination (40\%).

There was no correlation observed between CD4 count and neuropathic findings in our study, as these patients were selected within one month of diagnosis of HIV infection.

Evans et al reported prevalence neuropathy in preHAART patients to be $26 \%{ }^{(18)}$ and Cherry et al reported $42 \%$.(19) Subclinical neuropathy had been reported by Gastaut et al(20) in $40 \%$ of asymptomatic HIV seropositive patients. In advanced HIV infection or AIDS, the incidence of asymptomatic neuropathy was found to be $100 \% .{ }^{(20)}$ Chavanet et al found similar results in $10 \%$ and $53 \%$ of asymptomatic HIV positive subjects and AIDS patients respectively.(21)

The present study shows evidence of subclinical neuropathy in almost $69 \%$ of patients, the predominant type being distal axonal sensory neuropathy followed by axonal and demyelinating or mixed sensorimotor and motor neuropathy.

As the common diseases causing peripheral neuropathy and exposure to neurotoxins were excluded and as these patients were not on HAART(22), hence there is a likelihood of the neuropathy being caused by direct HIV affecting the peripheral nerves.

Careful assessment of the patients prior to the introduction of one of the potentially neurotoxic nucleoside analogues should be conducted to exclude those who are at risk of developing peripheral neuropathy with these agents or they may merely unmask a pre-existent subclinical distal neuropathy.

\section{LIMITATIONS}

Skin biopsy with estimation of intradermal nerve fiber density (IENFD) ( $<11$ fibres/mm) and morphological changes like increased density of axonal swellings(23) is a validated technique for the diagnosis of small fiber neuropathy, monitoring its progression(24) over time and for predicting the likelihood of condition developing in asymptomatic patients. Patients who had normal NCS in the study have not undergone skin biopsies.

\section{CONCLUSIONS}

The present study shows that the newly diagnosed HIV patients have neuropathy without symptoms. Electrophysiology studies have picked up neuropathy in twothirds of patients.

\section{REFERENCES}

1. WHO Global Health Observatory GHO 2014, UNAIDS 2015 factsheet 2015 statistics.

2. HIV Sentinel Surveillance 2012-13, a National Report by NACO India, New Delhi.

3. Fauci SAS, Lane HC. HIV disease-AIDS and related disorders. In: Fauci SAS, Braunwald E, Esselbacher RT, et al, eds. Harrison's principle of internal medicine. 14th edn. USA: McGraw Hill Co 1998:1791-855.

4. McArthur JC. Neurologic manifestations of AIDS. Medicine 1987;66(6):407-437.

5. Daroff RB, Fenichel GM, Jankovic J, et al. Bradley's neurology in clinical practice. $6^{\text {th }}$ edn. Vol. 1. Elsevier Saunders 2012:p. 2544.

6. Geraci AP, Simpson DM. Neurological manifestations of HIV-1 infection in HAART era. Compr Ther 2001;27(3):232-41.

7. Marra CM, Boutin P, Collier AC, et al. Screening for DSP in HIV infected person in research and clinical settings. Neurology 1998;51(6):1678-1681.

8. Tagliati M, Simpson DM, Grinnell J, et al. Peripheral nerve function in HIV infection: clinical, electrophysiologic and laboratory finding. Arch Neurol 1999;56(1):84-9.

9. Simpson DM, Tagliati M. Nucleoside analogue-associated peripheral neuropathy in HIV infection. J Acquire Immune Defici Syndr Retroviral 1995;9(2):153-61.

10. Hoke A, Cornblath DR. Peripheral neuropathies in HIV infection. Suppl Clin Neurophysiol 2004;57:195-210.

11. McArthur JC, Brew BJ, Nath A. Neurological complications of HIV infection. The Lancet Neur 2005;4(9):543-55. 
12. MorgelloS, Simpson D, Estanislao L, et al. HIV associated distal sensory polyneuropathy in the era of HAART. The Manhattan HIV brain bank. Arch Neurol 2004;61(4):54651.

13. Childs EA, Lyles RH, Selnes OA, et al. Plasma viral load and CD4 lymphocytes predict HIV-associated dementia and sensory neuropathy. Neurology 1999;52(3):607-13.

14. Cornblath DR, McArthur JC. Predominantly sensory neuropathy in patients with AIDS and AIDS-related complex. Neurology 1988;38(5):794-6.

15. Simpson DM, Kitch D, Evans SR, et al. HIV neuropathy natural history cohort study: assessment measures and risk factors. Neurology 2006;66(11):1679-87.

16. Polydefkis M, Yiannoutsos CT, Cohen BA, et al. Reduced IENFD in HIV associated sensory neuropathy. Neurol 2002;58(1):115-9.

17. Figg WD. Peripheral neuropathy in HIV patients after isoniazid therapy initiated. DICP 1991;25(1):100-1.

18. Evans D, Takuva S, Rassool M, et al. Prevalence of peripheral neuropathy in antiretroviral therapy naïve HIV positive patients and the impact on the treatment outcomes-a retrospective study from a large urban cohort in Johannesburg, South Africa. J Neurovirol 2012;18(3):162-7
19. Cherry CL, Skolacky RL, Lal L, et al. Antiretroviral use and other risks for HIV-associated neuropathies in an international cohort. Neurology 2006;66(6):867-73.

20. Gastaut JL, Gastaut JA, Pellissier JF, et al. Peripheral neuropathies in HIV infection. A prospective study of 56 patients. Rev Neurol 1989;145(6-7):451-9.

21. Chavanet PY, Giroud M, Lancon JP, et al. Altered peripheral nerve conduction in HIV patients. Cancer Detect Prev 1988;12(1-6):249-55.

22. Dalakas MC, Mora SC, Monzon LM. Mitochondrial alterations with mitochondrial DNA depletion in nerves of AIDS patients with PN induced by DDC. Lab Invest 2001;81(11):1537-44.

23. Lauria G, Cornblath DR, Johansson O, et al. EFNS guidelines on the use of skin biopsy in the diagnosis of peripheral neuropathy. Eur J Neurol 2015;12(10):74758.

24. Lauria G, Morbin M, Lombardi R, et al. Axonal swellings predict the degeneration of epidermal nerve fibres in painful neuropathies. Neurology 2003;61(5):631-6. 